DOI: http://dx.doi.org/10.5007/2175-8069.2016v13n28p3

\title{
Efeito de informações precedentes no comportamento assimétrico dos custos
}

\author{
Effect of precedent information on asymmetric cost behavior
}

Efectos de informaciones precedentes en el comportamiento asimétrico de los costos

\section{Laura Rodrigues Cardoso Ferreira}

Graduada em Ciências Contábeis pela Faculdade de Ciências Contábeis da Universidade Federal de Uberlândia (UFU)

Endereço: Av. João Naves de Ávila, n॰2121 - Bloco F - Sala 1F 215 - Campus Santa Mônica CEP: 38.400-902 - Uberlândia/MG - Brasil

E-mail: laura-rodrigues91@hotmail.com

Telefone: + (55) (34) 3218-1676 / 3291-5904

\section{Patrícia de Souza Costa}

Professora da Faculdade de Ciências Contábeis da Universidade Federal de Uberlândia (UFU) Doutora em Contabilidade e Controladoria pela Faculdade de Economia, Administração e Contabilidade da Universidade de São Paulo (FEA/USP).

Endereço: Av. João Naves de Ávila, n॰ 2121 - Bloco F - Sala 1F 215 - Campus Santa Mônica CEP: 38.400-902 - Uberlândia/MG - Brasil

E-mail: patricia.costa@ufu.br

Telefone: +(55) (34) 99155-9524 / 3291-5904

\section{Jéssica Rayse de Melo Silva Ávila}

Mestre em Contabilidade pelo Programa de Pós-Graduação em Ciências Contábeis da Universidade Federal de Uberlândia (UFU)

Endereço: Av. João Naves de Ávila, no 2121 - Bloco F - Sala 1F 215 - Campus Santa Mônica CEP: 38.400-902 - Uberlândia/MG - Brasil

E-mail: jessicarayse@hotmail.com

Telefone: + (55) (34) 99639-8509

Artigo recebido em 26/10/2015. Revisado por pares em 03/03/2016. Reformulado em 05/04/2016. Recomendado para publicação em 20/04/2016 por Sandra Rolim Ensslin (Editora Científica). Publicado em 14/06/2016. 


\title{
Resumo
}

$\mathrm{Na}$ literatura que trata dos sticky costs tem-se que os custos variam de maneira assimétrica em relação às variações nas vendas, podendo a variação precedente da receita afetar o comportamento dos custos. Dessa forma, o objetivo desta pesquisa é analisar se existe relação entre informações precedentes de vendas - crescimento histórico das vendas, a relação market-to-book e a volatilidade das vendas - e o comportamento assimétrico dos custos. A amostra da pesquisa é composta por 218 companhias abertas brasileiras com demonstrações financeiras publicadas no período de 1995-2013. Os resultados indicam que o crescimento histórico das vendas, a relação market-to-book e a volatilidade das vendas possuem relação com a assimetria dos custos, sugerindo que as variações precedentes da receita afetam o comportamento dos custos.

Palavras-chave: Custos assimétricos. Volatilidade das vendas. Relação market-to-book; Informações precedentes.

\begin{abstract}
In the sticky costs literature, it is mentioned that the costs may change asymmetrically when it relates to the changes in sales, can foregoing change of the revenue to affect the cost behavior. In this way, this study aims to analyze the existence between foregoing information's of sales - historical sales growth, the market-to-book relation and the volatility of sales- and the asymmetric cost behavior. The research sample is composed by 218 Brazilian publicly held companies with published financial statements between 1995-2013. The results suggest that the historical sales growth, the market-to-book relation and the volatility of sales are related to the costs asymmetry, suggesting that foregoing change of the revenue to affect the cost behavior.
\end{abstract}

Keywords: Asymmetric costs. Volatility of sales. Market-to-book relation. Preceding information.

\section{Resumen}

La literatura que aborda el tema de los sticky costs asume que los costos varían de forma asimétrica en relación con las variaciones de ventas, puede la variación precedente en las ventas afectar el comportamiento de los costos. Por lo tanto, el objetivo de esta investigación es analizar si existe una relación entre la información precedente de ventas - el crecimiento histórico de las ventas, la relación market-to-book y la volatilidad de las ventas - y el comportamiento asimétrico de los costos. La muestra está conformada por 218 compañías abiertas brasileras con Estados Financieros publicados del periodo 1995-2013. Los resultados indican que el crecimiento histórico de ventas, la relación market-to-book y la volatilidad de las ventas poseen relación con la asimetría de los costos, lo que sugiere que las variaciones precedentes en las ventas afectan al comportamiento de los costos.

Palabras claves: Costos asimétricos. Volatilidad de las ventas. Relación market-to-book. Informaciones precedentes. 


\section{Introdução}

O modelo tradicional de análise do comportamento dos custos considera, segundo Noreen (1991), que os custos variam proporcionalmente à oscilação do volume de operações, sem considerar a direção dessa variação. Entretanto, a linearidade dos custos em relação às atividades foi questionada por Noreen e Soderstrom (1994). Estes autores acreditavam que a suposição de assimetria é conflitante com os conceitos de economia em escala, em que o custo médio deve reduzir à medida que o volume aumenta. Os autores verificaram empiricamente que o custo marginal (alteração no custo total quando da variação na quantidade produzida) não é igual ao custo médio para a maioria das contas, rejeitando a hipótese de proporcionalidade. Em outra ocasião, Noreen e Soderstrom (1997) não encontraram evidências da não linearidade, pois verificaram que praticamente a totalidade do efeito nos custos ocorre em consonância com mudança no volume de atividades.

Por outro lado, Anderson, Banker e Janakiraman (2003), utilizando uma amostra de empresas americanas, comprovaram que os custos variam de maneira assimétrica em relação às variações nas receitas; ou seja, os custos aumentam com maior intensidade quando a receita aumenta do que no sentido oposto. Medeiros, Costa e Silva (2005) testaram os modelos de custos assimétricos propostos por Anderson, Banker e Janakiraman (2003) no contexto brasileiro. Os achados dessa pesquisa confirmaram a hipótese de que os custos das empresas brasileiras estudadas apresentam elasticidade assimétrica em relação a variações nas receitas, de forma que os custos aumentam com maior intensidade quando a receita aumenta, mas não variam na mesma proporção quando ela diminui. Em relação às empresas latino-americanas, Costa et al. (2013) concluíram que o comportamento das despesas de vendas, gerais e administrativas também é assimétrico em relação às mudanças na receita de vendas.

Anderson, Asdemir e Tripathy (2013) encontraram que a assimetria de custos está associada com a incerteza na demanda. Essa incerteza pode afetar as decisões dos gestores em relação ao comprometimento dos custos. Especificamente, esses autores identificaram que a assimetria de custos aumenta com o crescimento histórico das vendas e a relação market-tobook e diminui com a volatilidade histórica das vendas. Espera-se que os recursos comprometidos variem com o crescimento histórico das vendas, uma vez que um crescimento persistente pode aumentar a capacidade do gestor de planejar adequadamente a capacidade e os investimentos em custos fixos.

A relação market-to-book representa a expectativa dos investidores relacionada aos lucros anormais, podendo ser usada como proxy para a expectativa de magnitude e de persistência no crescimento das vendas. Uma vez que a volatilidade histórica das vendas prediz a volatilidade futura, os gestores podem reagir à volatilidade histórica controlando os investimentos em custos fixos. Com esses argumentos, Anderson, Asdemir e Tripathy (2013) identificaram que informações precedentes podem afetar o comportamento assimétrico dos custos.

A amostra da pesquisa de Anderson, Asdemir e Tripathy (2013) é composta por companhias abertas americanas, cujo país de origem baseia-se na common law, desenvolvido economicamente e com regras contábeis dissociadas das regras fiscais. Nesses países esperase que as informações contábeis possuam qualidade superior a daquelas de países com base na code law. O ambiente econômico, o sistema legal de cada país, a cultura das empresas, o sistema de governança corporativa e o nível de enforcement podem interferir na magnitude da assimetria de custos. Calleja, Steliaros e Thomas (2006) identificaram que os custos das 
companhias de países code law (França e Alemanha) são mais assimétricos do que aqueles de companhias de países common law (Estados Unidos e Reino Unido). Esses autores atribuem essa diferença ao sistema de governança corporativa e à fiscalização externa da atuação dos gestores quanto ao controle dos custos. Por outro lado, Banker, Byzalov e Chen (2013) não encontraram diferenças na assimetria de custos entre países de origem legalista e não legalista.

Diante desse contexto, o objetivo desta pesquisa é analisar se existe relação entre informações precedentes de vendas - crescimento histórico das vendas, a relação market-tobook e a volatilidade das vendas - e o comportamento assimétrico dos custos. A amostra da pesquisa é composta por 218 companhias abertas brasileiras com demonstrações financeiras publicadas no período de 1995-2013. Além das despesas de vendas, gerais e administrativas utilizadas no estudo de Anderson, Asdemir e Tripathy (2013) para representar os custos, este estudo analisou, também, o comportamento dos custos dos produtos vendidos e dos serviços prestados.

As companhias abertas brasileiras estão inseridas em um contexto de maior instabilidade econômica e política, sendo o Brasil um país em desenvolvimento e de origem legalista. Dessa forma, os resultados desta pesquisa podem ser divergentes daqueles encontrados por Anderson, Asdemir e Tripathy (2013), podendo contribuir para o desenvolvimento da teoria de assimetria de custos. Esses resultados podem ser úteis para gestores e acionistas analisarem como essas variáveis precedentes podem ser utilizadas na gestão estratégica de custos.

O trabalho está dividido em quatro seções; após esta introdução é apresentado o referencial teórico sobre a assimetria de custos e a incerteza na demanda, bem como são estipuladas as três hipóteses a serem testadas no estudo. Os resultados da pesquisa e a discussão dos resultados são ilustrados na terceira seção. Na última seção são apresentadas as considerações finais.

\section{Referencial Teórico}

A plataforma teórica foi desenvolvida de modo a abarcar os conceitos que podem consubstanciar uma possível relação entre os custos assimétricos e as informações precedentes sobre a receita.

\subsection{Assimetria de Custos}

A assimetria de custos é consistente com o argumento de que os gestores tendem a ajustar recursos deliberadamente, em resposta a mudanças no volume (COSTA et al., 2013). Desse modo, quando há diminuição nas vendas da organização, os gerentes podem adiar propositadamente reduções de recursos comprometidos até que eles possam ter mais certeza sobre a permanência de um declínio na demanda (COSTA et al., 2013). Eles também tendem a manter os recursos não utilizados para evitar consequências pessoais na redução de despesas. Além disso, pode haver um intervalo de tempo entre a decisão de reduzir os recursos comprometidos e a realização da redução de custos (ANDERSON; BANKER; JANAKIRAMAN, 2003). Para Medeiros, Costa e Silva (2005), os gerentes que têm conhecimento a respeito de como os custos se comportam possuem melhores condições de se 
preparar para a tomada de decisões, de acordo com a trajetória dos custos em diversas situações operacionais, pois, assim, podem planejar melhor suas atividades e, consequentemente, o lucro.

As pesquisas que consideram a não linearidade dos custos em relação às atividades desenvolveram-se mais enfaticamente a partir do estudo de Noreen e Soderstrom (1994), que buscaram validar empiricamente se os custos eram estritamente proporcionais ao volume de atividade de um setor específico, o hospitalar. Por meio de análise cross-section, os autores verificaram que, para aquela amostra, o custo médio por unidade aumentou os custos marginais em aproximadamente $40 \%$ para alguns setores, chegando a $100 \%$ para outros, rejeitando a hipótese de proporcionalidade entre custos e atividades. Em outra ocasião, Noreen e Soderstrom (1997) lançaram mão de análises de séries temporais para analisar o comportamento dos custos gerais também no setor hospitalar, e concluíram que as projeções mais precisas de mudanças nos custos assumem que o custo não muda, ao invés de assumirem que o custo muda em proporção ao nível de atividade.

Complementando os achados de Noreen e Soderstrom (1997), Anderson, Banker e Janakiraman (2003) chamaram de sticky costs aos custos que estão ligados à não ocorrência de variação semelhante, em caso de elevação ou diminuição no volume de atividades, especificamente de vendas. Esses custos podem ser traduzidos por custos rígidos, custos com variação assimétrica ou custos com elasticidade assimétrica (MEDEIROS; COSTA; SILVA, 2005). A amostra da pesquisa de Anderson, Banker e Janakiraman (2003) é composta por dados financeiros de 7.629 empresas norte-americanas, por um período de mais de 20 anos. Os autores analisaram o que seria um modelo alternativo de comportamento de custos, onde ocorrem os sticky costs, argumentando que uma diversidade de combinações de recursos (marketing, comissões de vendas, frete, propaganda, salários do pessoal administrativo, etc.) é necessária para dar suporte às atividades de vendas, gerais e administrativas. Eles explicam que as despesas com atividades de vendas, gerais e administrativas (VGA) são mais difíceis de gerenciar do que os custos gerados nas atividades de produção de bens e serviços, e incorrem em períodos derivados de diversos contratos, incluindo aluguel, leasing e empregados permanentes e temporários. Para esses autores, as VGA não variam necessariamente com o volume de atividades e precisam ser gerenciadas através da combinação de recursos fixos e variáveis, a fim de suportar o nível de vendas.

Anderson, Banker e Janakiraman (2003) comprovaram que os custos possuem comportamento assimétrico em relação à receita, uma vez que eles aumentam em média $0,55 \%$ para um incremento de $1 \%$ na receita líquida de vendas, mas diminuem somente $0,35 \%$ para uma redução de $1 \%$ na receita líquida de vendas. Esses autores atribuem essa assimetria a diversos possíveis fatores, como a ocorrência de conflitos de agência, os custos de ajuste psicológico (normalmente identificado na dispensa de funcionários, devido ao impacto negativo sobre sua imagem pessoal), e o tempo gasto para certificar-se de que não se trata de um impacto transitório no volume de atividade da empresa em relação ao mercado. A relutância de dispensar funcionários ocorre pelo fato de que os gestores possuem a expectativa das vendas aumentarem novamente e não necessitar de chegar à decisão de demitir empregados. Os custos de agência são custos incorridos pela empresa por causa de decisões tomadas pelos gerentes com base na maximização de seus interesses próprios, e não na perspectiva de criar valor para os acionistas da empresa (MEDEIROS; COSTA; SILVA, 2005). Ainda, a necessidade de tempo para confirmar a tendência de redução do volume de 
atividade também é algo requerido pelo gestor, que demanda esse tempo para avaliar melhor a sua decisão e a probabilidade de que as vendas continuem em baixo volume.

Subramaniam e Weidenmier (2003) analisaram os custos assimétricos em um conjunto de dados de 22 anos da Compustat durante o período 1979 a 2000. Os resultados do estudo apontam que as despesas com vendas, gerais e administrativas e o custo de produtos possuem comportamento rígido, individualmente e em conjunto, para todas as empresas analisadas. Outro achado é que os custos também são assimétricos quando a receita varia em mais de $10 \%$. Quando a receita aumenta em mais de $10 \%$, os gestores comprometem mais recursos na empresa, de forma a ampliar a capacidade; mas quando as receitas diminuem em mais de $10 \%$, os gestores não são capazes ou não desejam reduzir a capacidade da empresa, fazendo com que os custos apresentem um comportamento pegajoso.

O comportamento assimétrico dos custos foi confirmado em amostras de companhias de vários países, como Estados Unidos, Brasil, Japão, Inglaterra, França, Alemanha e outros. Foram descritas diferenças em países de regime code law e regime common law. Por exemplo, na pesquisa feita por Calleja, Steliaros e Thomas (2006), os autores identificaram que a variação dos custos na França e na Alemanha (países de regime code law) é mais assimétrica do que a variação dos custos na Inglaterra e nos Estados Unidos (países de regime common law); nos países legalistas os custos operacionais aumentam, em média, $0,97 \%$ ao aumento de $1 \%$ na receita, mas reduzem apenas $0,91 \%$ por $1 \%$ por receita diminuída. Isso sugere que diversas variáveis, como a localização, o sistema jurídico do país de origem, o tamanho da companhia e número de funcionários, a governança corporativa adotada e a supervisão gerencial podem afetar a amplitude com que a companhia é afetada pela assimetria dos custos.

Para Medeiros, Costa e Silva (2005), o conhecimento do comportamento dos custos não é importante somente para gestores, também para usuários externos que avaliam o desempenho da empresa. Um exemplo dado por esses autores é a metodologia dos analistas financeiros, na qual o procedimento comum envolve a comparação de itens de despesas como um percentual das receitas líquidas de vendas entre empresas ou dentro da mesma empresa ao longo do tempo. Para esses autores, essa análise pode ser incorreta, se o comportamento dos custos em relação à receita não for observado e compreendido pelos analistas. Nessa perspectiva, investigar o comportamento dos custos é importante para o controle dos processos, maximização dos lucros, melhoria contínua e aumento do nível competitivo (ANDERSON; BANKER; JANAKIRAMAN, 2003; DUARTE et al., 2013).

Via e Perego (2013) afirmam que a análise do comportamento dos custos é uma questão crítica para apoiar decisões de gestão; porém, essa classificação tradicional da relevância é apenas para a magnitude da mudança, e não à sua direção. A validade teórica deste tipo de classificação está estritamente ligada ao horizonte de tempo e fatores contextuais devido ao ambiente econômico.

\subsection{Volatilidade, incerteza, informações precedentes e custos assimétricos}

O conhecimento gerado pelo estudo da assimetria de custos pode influenciar os gestores, formando forte relação com as expectativas de demandas futuras pelos produtos de suas companhias (KAMA; WEISS, 2013). Anderson, Asdemir e Tripathy (2013) vincularam assimetria de custos com variáveis precedentes que capturam informações sobre 0 crescimento da demanda e volatilidade das vendas. Nessa análise, foram usadas duas 
variáveis para capturar informações sobre o crescimento da demanda: o crescimento histórico de vendas e o valor de mercado da empresa. A volatilidade histórica de vendas foi utilizada como uma medida de volatilidade da demanda, e as expectativas eram de que as variáveis precedentes, histórico de crescimento de vendas e volatilidade fossem relevantes na avaliação do comportamento assimétrico dos custos, pois esses dados representam o ambiente de demanda nos termos do qual a tomada de decisão e sistemas de controle da empresa estão envolvidos.

A variável antecedente de valor de mercado deve ser um dado considerável para investidores, por se tratar de um indicador comum usado para avaliar o possível desenvolvimento das vendas futuras. De acordo com Anderson, Asdemir e Tripathy (2013), se os investimentos em recursos não correspondem à incerteza da demanda, uma empresa com crescimento persistente deve adotar mecanismos de controle que favoreçam recursos comprometidos, e uma empresa de alta volatilidade deve selecionar mecanismos que beneficiem recursos flexíveis. No entanto, para os autores, algumas empresas podem ser diferentes das outras por adotarem modelos de produção que as levam a maior volatilidade de vendas ou crescimento mais persistente.

A volatilidade no crescimento da receita de vendas (persistência das vendas) pode interferir no grau de investimento dos gestores em recursos comprometidos (ANDERSON; ASDEMIR, TRIPATHY, 2013). Se as vendas tendem a não ter altas variações nos períodos futuros, visualizando o histórico que ela possui, o gestor pode prever, com mais facilidade, o quanto pode investir na empresa. Assim, eles investem mais em ativos fixos, revisão de sistemas operacionais e gastos com pessoal, por exemplo, quando a empresa possui histórico de persistência no crescimento das vendas. Dessa forma, tem-se a primeira hipótese de pesquisa:

$\mathrm{H}_{1}$ : A assimetria de custos diminui com a magnitude e a persistência do crescimento histórico da receita.

Normalmente, os recursos comprometidos que conduzem ao comportamento assimétrico dos custos são recursos de longo prazo. Os gestores que investem em tais recursos podem desenvolver expectativas de longo prazo para as receitas. A avaliação do mercado de capitais fornece uma indicação da expectativa de crescimento dos investidores, e pode ser usada como proxy para a expectativa de crescimento das vendas no longo prazo (ANDERSON; ASDEMIR, TRIPATHY, 2013). A taxa market-to-book indica a expectativa dos investidores de lucros anormais. Isso reflete tanto a magnitude quanto expectativa de persistência no crescimento das vendas. Gestores com maior expectativa de vendas investirão mais em recursos comprometidos, a fim de obter vantagem competitiva. Uma vez que a assimetria de custos está associada positivamente com maior mix de recursos comprometidos e flexíveis, é esperada uma relação positiva entre o comportamento assimétrico dos custos e a relação market-to-book. Assim, a segunda hipótese de pesquisa é:

$\mathrm{H}_{2}$ : A assimetria de custos diminui com a magnitude da relação market-to-book.

Os gestores que enfrentam forte volatilidade de vendas da empresa estão menos dispostos a se comprometer com contratos que aumentam a capacidade de recursos, uma vez que tais contratos criariam uma incompatibilidade entre as flutuações da receita líquida e o 
comportamento dos custos, aumentando o risco operacional. Assim, na medida em que a volatilidade passada prevê a volatilidade futura, os gestores reagiriam às vendas antecedentes por meio da construção de flexibilidade nos componentes das despesas de vendas, gerais e administrativas da cadeia de valor, utilizando menos recursos financeiros.

H3: A assimetria de custos aumenta com a volatilidade histórica das vendas.

As hipóteses $\mathrm{H}_{1}, \mathrm{H}_{2}$ e $\mathrm{H}_{3}$ são baseadas nas diferenças na incerteza da demanda em um amplo conjunto de empresas.

\section{Metodologia da Pesquisa}

A metodologia utilizada para elaboração desta pesquisa é do tipo descritiva com abordagem quantitativa. A amostra da pesquisa consiste em dados anuais de companhias abertas brasileiras no período de 1995-2013. Da amostra inicial, composta por 394 companhias, foram excluídas aquelas pertencentes ao setor Finanças, Seguros e Fundos, restando 358 empresas. Foram, também, excluídas 140 empresas que não tiveram nenhum dado divulgado na base de dados Economática ${ }^{\mathrm{TM}}$ em todo o período de estudo, e aquelas que não tiveram dados de receita líquida divulgados em mais de 7 anos durante o já mencionado período de estudo. Assim, a amostra final da pesquisa é composta por 218 companhias abertas brasileiras.

O modelo proposto por Anderson, Banker e Janakiraman (2003) é utilizado nesta pesquisa para identificar a variação assimétrica dos custos em relação à receita de vendas (Equação 1).

$$
\log \left[\frac{V G A_{i, t}}{V G A_{i, t-1}}\right]=\beta_{0}+\beta_{1} \log \left[\frac{R L V_{i, t}}{R L V_{i, t-1}}\right]+\beta_{2} * D u m m y_{i, t} * \log \left[\frac{R L V_{i, t}}{R L V_{i, t-1}}\right]+\varepsilon_{i t}
$$

A variável VGA representa os custos e é constituída pelas despesas de vendas, gerais e administrativas. $\mathrm{O}$ modelo apresentado nas Equações 1 e 2 também foram testados utilizando a variável dependente VAGCPV, composta pelas despesas de vendas, gerais, administrativas e pelos custos dos produtos vendidos e serviços prestados. A variável RLV é igual à receita líquida de vendas. A variável dummy tem valor 1 quando a receita líquida da empresa $i$, no período $t$, é menor do que a receita líquida no período $t-1$. Tendo em vista que o valor da dummy é igual a 0 quando a receita aumenta, o coeficiente $\beta 1$ mede o percentual de aumento nos custos em relação a um aumento de $1 \%$ na receita. Devido ao valor 1 da dummy quando a receita diminui, a soma dos coeficientes $(\beta 1+\beta 2)$ mede o percentual de redução nos custos em relação a uma redução de $1 \%$ na receita. Se os custos possuem variação assimétrica, a variação dos custos em relação ao aumento da receita deve ser maior que a variação em relação à redução de receita.

Para testar as hipóteses $\mathrm{H}_{1}, \mathrm{H}_{2}$ e $\mathrm{H}_{3}$ utilizou-se o modelo expandido de Anderson, Asdemir e Tripathy (2013), apresentado na Equação 2. A utilização desse modelo irá possibilitar a comparação dos resultados desta pesquisa, composto por dados de companhias

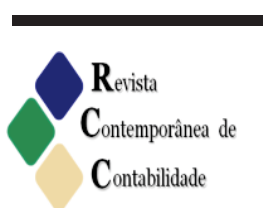

ISSN 2175-8069, UFSC, Florianópolis, v. 13, n. 28, p. 03-18, jan./abr. 2016 
abertas brasileiras (de origem code law), com aqueles da pesquisa de Anderson, Asdemir e Tripathy (2013), cujos dados são de companhias de países common law.

$$
\begin{aligned}
\log \left[\frac{V G A_{i, t}}{V G A_{i, t-1}}\right]= & \beta_{0}+\beta_{1} \log \left[\frac{R L V_{i, t}}{R L V_{i, t-1}}\right]+\beta_{2} * \text { Dummy }_{i, t} * \log \left[\frac{R L V_{i, t}}{R L V_{i, t-1}}\right] \\
& +\beta_{3} * \operatorname{CRESC}_{i, t-1} * \text { Dummy }_{i, t} * \log \left[\frac{R L V_{i, t-1}}{R L V_{i, t-1}}\right] \\
& +\beta_{4} * \operatorname{VOLAT}_{i, t-1} * \text { Dummy }_{i, t} * \log \left[\frac{R L V_{i, t-1}}{R L V_{i, t-1}}\right]+\varepsilon_{i, t}
\end{aligned}
$$

A variável CRESC representa a média histórica do crescimento das vendas nos últimos cinco anos. A variável VOLAT é o desvio padrão da receita de vendas (dividido pelo ativo total) nos últimos cinco anos. O modelo também foi testado substituindo a variável CRESC pela variável MTB (relação market-to-book da empresa $i$ no período $t$, dividida pela relação market-to-book da empresa $i$ no período $t$-1). A relação market-to-book resulta da divisão do valor de mercado da empresa pelo valor do patrimônio líquido.

Os dados da pesquisa foram coletados na base de dados Economática ${ }^{\mathrm{TM}}$, e conferidos por amostragem com os dados apresentados pela BM\&FBOVESPA. Foram testados os três modelos de dados em painel, a saber: efeitos comuns, efeitos fixos e efeitos aleatórios. Para definição do modelo mais adequado para dados em painel a ser utilizado na pesquisa foram realizados os testes de adequação do modelo estatístico de Breusch-Pagan, Chow e Hausman. Os resultados destes testes indicaram que o modelo de efeitos comuns (pooled) é mais adequado para os modelos especificados nas Equações 1 e 2. São apresentados, também, os resultados para os modelos fixos e aleatórios para efeito de comparação.

\section{Análise dos Resultados}

Na Tabela 1 é apresentada a estatística descritiva com o número de observações, a

\begin{tabular}{|c|c|c|c|c|c|c|c|c|}
\hline Variáveis & N. Obs & Média & $\begin{array}{l}\text { Desvio } \\
\text { Padrão }\end{array}$ & Mínimo & $\begin{array}{c}\text { Percentil } \\
25 \\
\end{array}$ & Mediana & $\begin{array}{c}\text { Percentil } \\
75\end{array}$ & Máximo \\
\hline VGA & 2.664 & 0,087 & 0,412 & $-4,752$ & $-0,007$ & 0,097 & 0,211 & 4,560 \\
\hline VGACPV & 2.701 & 0,102 & 0,443 & $-7,110$ & 0,012 & 0,108 & 0,215 & 5,963 \\
\hline RLV & 2.674 & 0,107 & 0,475 & $-8,762$ & 0,013 & 0,111 & 0,226 & 10,262 \\
\hline DRLV & 2.674 & $-0,068$ & 0,326 & $-8,762$ & 0,000 & 0,000 & 0,000 & 0,000 \\
\hline CRESC & 2.386 & $-0,082$ & 0,433 & $-10,447$ & 0,000 & 0,000 & 0,000 & 0,433 \\
\hline МТB & 2.036 & $-0,063$ & 0,614 & $-13,343$ & 0,000 & 0,000 & 0,000 & 9,418 \\
\hline VOLAT & 2.401 & $-0,015$ & 0,128 & $-3,576$ & 0,000 & 0,000 & 0,000 & 0,000 \\
\hline
\end{tabular}
média, desvio padrão e os valores máximos e mínimos das variáveis do estudo.

Tabela 1 - Estatística Descritiva

Fonte: Elaborada pelas autoras (2016).

A média obtida para a variável VGA, que representa as despesas de venda, gerais e administrativas, foi de 0,087 , mas com alto desvio padrão, comprovado pelos valores de 
mínimo e máximo, que se distanciam significativamente da média. Características semelhantes foram encontradas quando o CPV é somado ao VGA (VGACPV) e para a RLV. Para a variável crescente, a média é negativa e resultados semelhantes foram encontrados para valor de mercado e volatilidade.

$\mathrm{Na}$ Tabela 2 é apresentada a correlação entre as variáveis. Foram utilizados os coeficientes de Spearman, pois os dados não apresentam distribuição normal.

Tabela 2 - Coeficiente de correlação para as variáveis do estudo

\begin{tabular}{l|ccccccc}
\hline Variáveis & VGA & VGACPV & RLV & DRLV & CRESC & MTB & VOLAT \\
\hline VGA & 1,000 & & & & & & \\
VGACPV & 0,797 & 1,000 & & & & & \\
RLV & 0,508 & 0,786 & 1,000 & & & & \\
DRLV & 0,329 & 0,542 & 0,764 & 1,000 & & & \\
CRESC & 0,296 & 0,506 & 0,743 & 0,861 & 1,000 & & \\
MTB & 0,150 & 0,207 & 0,235 & 0,284 & 0,285 & 1,000 & \\
VOLAT & 0,215 & 0,402 & 0,552 & 0,653 & 0,561 & 0,140 & 1,000 \\
\hline
\end{tabular}

OBS.: Todas as variáveis significativas ao nível de $99 \%$ de confiança.

Fonte: Elaborada pelas autoras (2016).

Observa-se que a variável CRESC apresenta correlação significativa e positiva com a variável VGA, indicando que o aumento das despesas de vendas, gerais e administrativas está associado a um aumento no crescimento histórico das vendas, o que oferece indícios de aceitação da hipótese $\mathrm{H}_{1}$. O mesmo ocorre entre MTB, sugerindo que a hipótese $\mathrm{H} 2$ está adequadamente especificada. O comportamento das variáveis, quando observada a variável VGACPV, é semelhante, sugerindo que a inclusão dos custos dos produtos vendidos e serviços prestados não influencia na direção dos sinais das variações.

De acordo com a Tabela 3 , o coeficiente $\beta_{1}$, mede a elasticidade dos custos em relação à receita de vendas e possui valor significativo de 0,482 , indicando que os custos aumentam $0,482 \%$ para um aumento de $1 \%$ da receita de vendas. Este resultado é condizente com aquele encontrados por Medeiros, Costa e Silva (2005), Marques et al. (2014) e Anderson, Asdemir e Tripathy (2013). Esses autores encontraram um aumento de $0,549 \%, 0,560 \%$ e $0,645 \%$ na VGA, respectivamente, para cada $1 \%$ de aumento nas receitas.

Assim como nos estudos de Medeiros, Costa e Silva (2005), Marques et al. (2014) e Anderson, Asdemir e Tripathy (2013), o coeficiente $\beta_{2}$ apresentou sinal negativo. Entretanto, a interpretação desta variável tem mais sentido econômico quando é somada ao coeficiente $\beta_{1}$, fornecendo subsídios para verificação da assimetria dos custos. $O$ resultado de $\beta_{1}+\beta_{2}=0,319$ sugere que os custos reduziram $0,319 \%$, diante de uma queda de $1 \%$ na receita de vendas, corroborando com os resultados de Medeiros, Costa e Silva (2005), Marques et al. (2014) e Anderson, Asdemir e Tripathy (2013), que verificaram que os custos reduziram 0,30\%, 0,45\% e $0,455 \%$, respectivamente, para uma queda de $1 \%$ na RLV. Para a VGA, o modelo apresentou $\mathrm{R}^{2}$ de 0,266 , indicando que aproximadamente $27 \%$ da variação dos dados são explicados pelo método aplicado. 
Tabela 3 - Coeficientes estimados de VGA e VGA-CPV - Equação 1

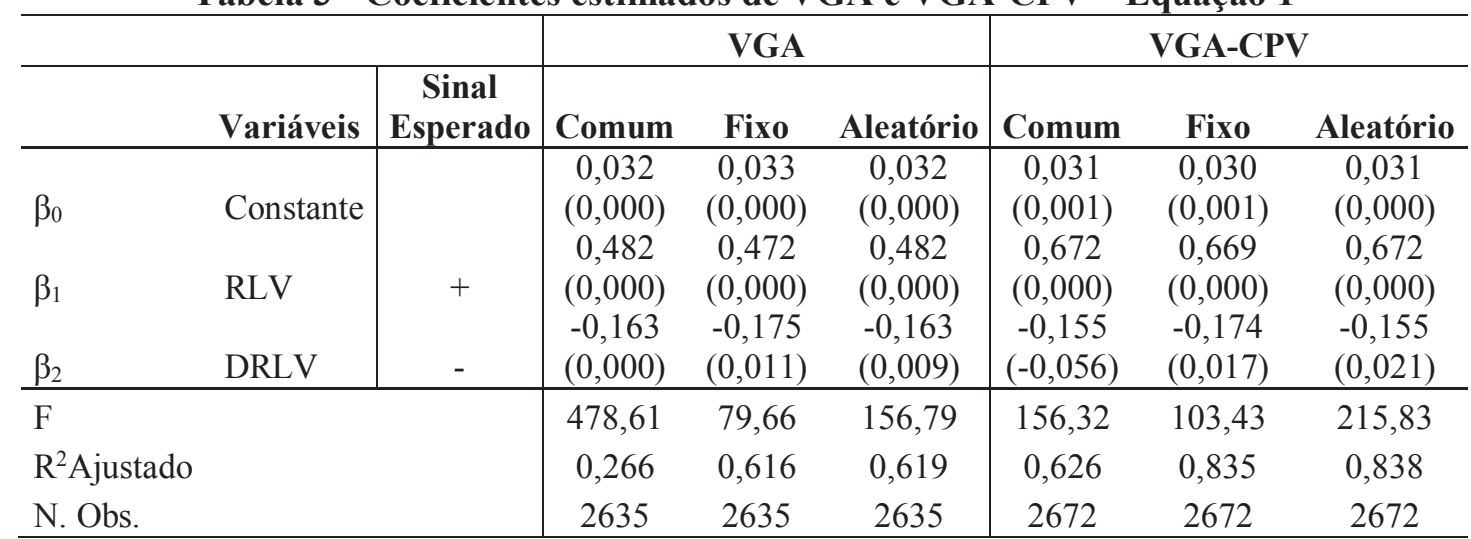

Fonte: Elaborada pelas autoras (2016).

Percebe-se que o modelo estimado, quando a variável dependente é a VGA-CPV, também apresentou indícios de assimetria de custos. O coeficiente $\beta_{1}$ encontrado foi de 0,672 , sugerindo que os custos aumentam $0,672 \%$ para o aumento de $1 \%$ da receita de vendas. $\mathrm{O}$ coeficiente $\beta_{2}$ é $-0,155$; assim, $\beta_{1}+\beta_{2}=0,517$, indicando que os custos reduziram $0,517 \%$ para uma queda de $1 \%$ na receita de vendas. Para o modelo com a variável VGA-CPV, o $\mathrm{R}^{2}$ é significativamente maior, indicando que aproximadamente $63 \%$ da variação dos dados são explicados pelo modelo aplicado. Dessa forma, percebe-se que tanto as despesas de vendas, gerais e administrativas, quanto os custos dos produtos vendidos e serviços prestados apresentam assimetria em relação à receita de vendas. Porém, a assimetria de custos é maior quando a variável dependente é VGA $(0,482-0,319=0,163)$, do que a assimetria para a variável dependente é VGACPV $(0,672-0,517=0,155)$, sugerindo que os gestores demoram mais para cortar as despesas de vendas, gerais e administrativas diante de uma redução na receita líquida.

A Tabela 4 demonstra os coeficientes estimados ao se considerar, também, os parâmetros crescimento e volatilidade das vendas. $\mathrm{O}$ crescimento e a volatilidade interagem entre si no ajuste de expectativas com relação à demanda da receita de vendas (ANDERSON; ASDEMIR; TRIPATHY, 2013).

Para o modelo da Equação 2, considerando a variável dependente VGA, o coeficiente $\beta_{1}$ possui valor significativo de 0,471 , indicando que os custos aumentam $0,471 \%$ para um aumento de $1 \%$ da receita de vendas (Tabela 4 ).

O coeficiente $\beta_{2}$ também apresentou sinal negativo. A soma de $\beta_{1}$ e $\beta_{2}$ foi de 0,349 , ou seja, os custos reduziram $0,349 \%$ para uma queda de $1 \%$ na receita de vendas, sugerindo assimetria de custos. Estes resultados permanecem condizentes com aqueles encontrados por Medeiros, Costa e Silva (2005), Marques et al. (2014) e Anderson, Asdemir e Tripathy (2013).

O sinal encontrado para o coeficiente $\beta 3(-0,655)$, negativo e significante, sugere que os custos assimétricos diminuem à medida que o crescimento histórico de vendas aumenta (é mais persistente), suportando a hipótese H1. Estes resultados são semelhantes àqueles encontrados por Anderson, Asdemir e Tripathy (2013), sugerindo que os gestores conseguem prever os recursos (evitando a assimetria) de maneira mais adequada quando as empresas apresentam histórico de persistência no crescimento das vendas. 
Tabela 4 - Coeficientes estimados de VGA e VGA-CPV - Equação 2

\begin{tabular}{|c|c|c|c|c|c|c|c|c|}
\hline & \multirow[b]{2}{*}{ Variáveis } & \multirow[b]{2}{*}{$\begin{array}{c}\text { Sinal } \\
\text { Esperado }\end{array}$} & \multicolumn{3}{|c|}{ Variável dependente VGA } & \multicolumn{3}{|c|}{ Variável dependente VGACPV } \\
\hline & & & Comum & Fixo & Aleatório & Comum & Fixo & Aleatório \\
\hline \multirow{3}{*}{$\beta_{0}$} & \multirow{3}{*}{ Constante } & \multirow{5}{*}{+} & 0,030 & 0,030 & 0,030 & 0,023 & 0,022 & 0,023 \\
\hline & & & $(0,008)$ & $(0,005)$ & $(0,001)$ & $(0,000)$ & $(0,000)$ & $(0,000)$ \\
\hline & & & 0,471 & 0,461 & 0,471 & 0,694 & 0,694 & 0,694 \\
\hline \multirow[t]{2}{*}{$\beta_{1}$} & \multirow[t]{2}{*}{ RLV } & & $(0,000)$ & $(0,000)$ & $(0,000)$ & $(0,000)$ & $(0,000)$ & $(0,000)$ \\
\hline & & & $-0,122$ & $-0,100$ & $-0,122$ & $-0,192$ & $-0,185$ & $-0,192$ \\
\hline \multirow[t]{2}{*}{$\beta_{2}$} & \multirow[t]{2}{*}{ DRLV } & \multirow[t]{2}{*}{-} & $(0,402)$ & $(0,497)$ & $(0,389)$ & $(0,000)$ & $(0,000)$ & $(0,000)$ \\
\hline & & & $-0,021$ & $-0,051$ & $-0,021$ & $-0,017$ & $-0,044$ & $-0,017$ \\
\hline \multirow[t]{2}{*}{$\beta_{3}$} & \multirow[t]{2}{*}{ CRESC } & \multirow[t]{2}{*}{-} & $(0,655)$ & $(0,432)$ & $(0,690)$ & $(0,413)$ & $(0,051)$ & $(0,413)$ \\
\hline & & & $-0,045$ & $-0,024$ & $-0,045$ & 0,110 & 0,112 & 0,110 \\
\hline$\beta_{4}$ & VOLAT & + & $(0,849)$ & $(0,923)$ & $(0,839)$ & $(0,031)$ & $(0,025)$ & $(0,030)$ \\
\hline $\mathrm{F}$ & & & 26,23 & 15,97 & 65,64 & 782,63 & 653,75 & 3130,51 \\
\hline $\mathrm{R}^{2}$ Ajustado & & & 0,211 & 0,49 & 0,507 & 0,568 & 0,773 & 0,785 \\
\hline N. Obs. & & & 2362 & 2362 & 2362 & 2384 & 2384 & 2384 \\
\hline
\end{tabular}

Fonte: Elaborada pelas autoras (2016).

Todavia, diferentemente do resultado encontrado por Anderson, Asdemir e Tripathy (2013), o coeficiente da variável VOLAT foi negativo e significante, sugerindo que os custos assimétricos diminuem à medida que a volatilidade das vendas aumenta, o que contraria a hipótese $\mathrm{H}_{3}$. Em outras palavras, o resultado sugere que os gestores que enfrentam forte volatilidade de vendas estão mais dispostos a se comprometer com contratos incompatíveis com as flutuações da receita líquida e do comportamento dos custos, aumentando, consequentemente, o risco operacional, ou possuem dificuldades na gestão dos custos quando expostos à volatilidade das vendas. Para a VGA deste modelo, o $\mathrm{R}^{2}$ foi menor, indicando que aproximadamente $21 \%$ da variação dos dados são explicados pelo modelo aplicado.

Já o coeficiente estimado $\beta_{1}$, considerando a variável dependente VGACPV na Equação 2, foi de 0,694, sugerindo que os custos aumentam 0,694\% para o aumento de $1 \%$ da receita de vendas. $O$ coeficiente $\beta_{2}$ de $-0,192$ é significante e negativo. Desta forma, $\beta_{1+} \beta_{2}=$ 0,502 , indicando que os custos reduziram $0,502 \%$ para uma queda de $1 \%$ na receita de vendas. Estes resultados também sugerem a assimetria de custos.

Quando a variável dependente é VGACPV (Tabela 4), os sinais encontrados para os coeficientes $\beta_{3}$ e $\beta_{4}$ são semelhantes àqueles do estudo de Anderson, Asdemir e Tripathy (2013), sugerindo que a assimetria dos custos diminui à medida que o crescimento histórico de vendas e a volatilidade nas vendas aumenta. Diante desse resultado, as hipóteses $\mathrm{H}_{1}$ e $\mathrm{H}_{3}$ não são rejeitadas. Além disso, para o modelo com a variável VGACPV, o $\mathrm{R}^{2}$ é significativamente maior, indicando que aproximadamente $67 \%$ da variação dos dados são explicados pelo modelo aplicado (Tabela 4).

De acordo com esses resultados (Tabela 4), tem-se que os gestores de empresas com histórico de volatilidade na receita líquida conseguem reduzir a assimetria dos custos dos produtos vendidos e serviços prestados, mas não conseguem essa mesma redução nas despesas de vendas, gerais e administrativas. Esses resultados diferem daqueles encontrados por Anderson, Asdemir e Tripathy (2013) para uma amostra de empresas americanas (origem common law), sugerindo que os gestores de países code law (como ocorre no Brasil) podem demorar a cortar despesas quando a empresa apresenta volatilidade das vendas.

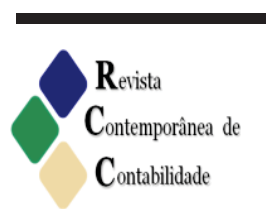


Para a definição do modelo mais adequado a ser utilizado para a Equação 2, considerando a variável MTB no lugar da variável CRESC, também foram realizados os testes de adequação do modelo estatístico de Breusch-Pagan, Chow e Hausman. Os resultados destes testes indicaram que, para o parâmetro em valor de mercado (market-tobook) e volatilidade, é mais adequado o modelo aleatório. Os resultados são apresentados na Tabela 5. São apresentados, também, os resultados para os modelos fixos e aleatórios para efeito de comparação.

Para o modelo com variável dependente VGA (Tabela 5), o coeficiente $\beta_{1}$ possui valor significativo de 0,451 , indicando que os custos aumentam $0,451 \%$ para um aumento de $1 \%$ da receita de vendas. $O$ coeficiente $\beta_{2}$ também obteve sinal negativo, como no estudo Anderson, Asdemir e Tripathy (2013), e o resultado da soma de $\beta_{1}$ e $\beta_{2}$ foi de 0,296 , ou seja, os custos reduziram apenas $0,296 \%$ para uma queda de $1 \%$ na receita de vendas, implicando na assimetria de custos.

Percebe-se que o coeficiente estimado, quando a variável dependente é VGACPV, é também assimétrico, pois o $\beta_{1}$ encontrado foi de 0,693 , sugerindo que os custos aumentam $0,693 \%$ para o aumento de $1 \%$ da receita de vendas, e o coeficiente $\beta_{2}$ é $-0,245$; assim, $\beta_{1}+\beta_{2}=$ 0,448 , indicando que os custos reduziram $0,448 \%$ para uma queda de $1 \%$ na receita de vendas.

Tabela 5 - Coeficientes Estimados de VGA e VGACPV - Equação 2 (MTB)

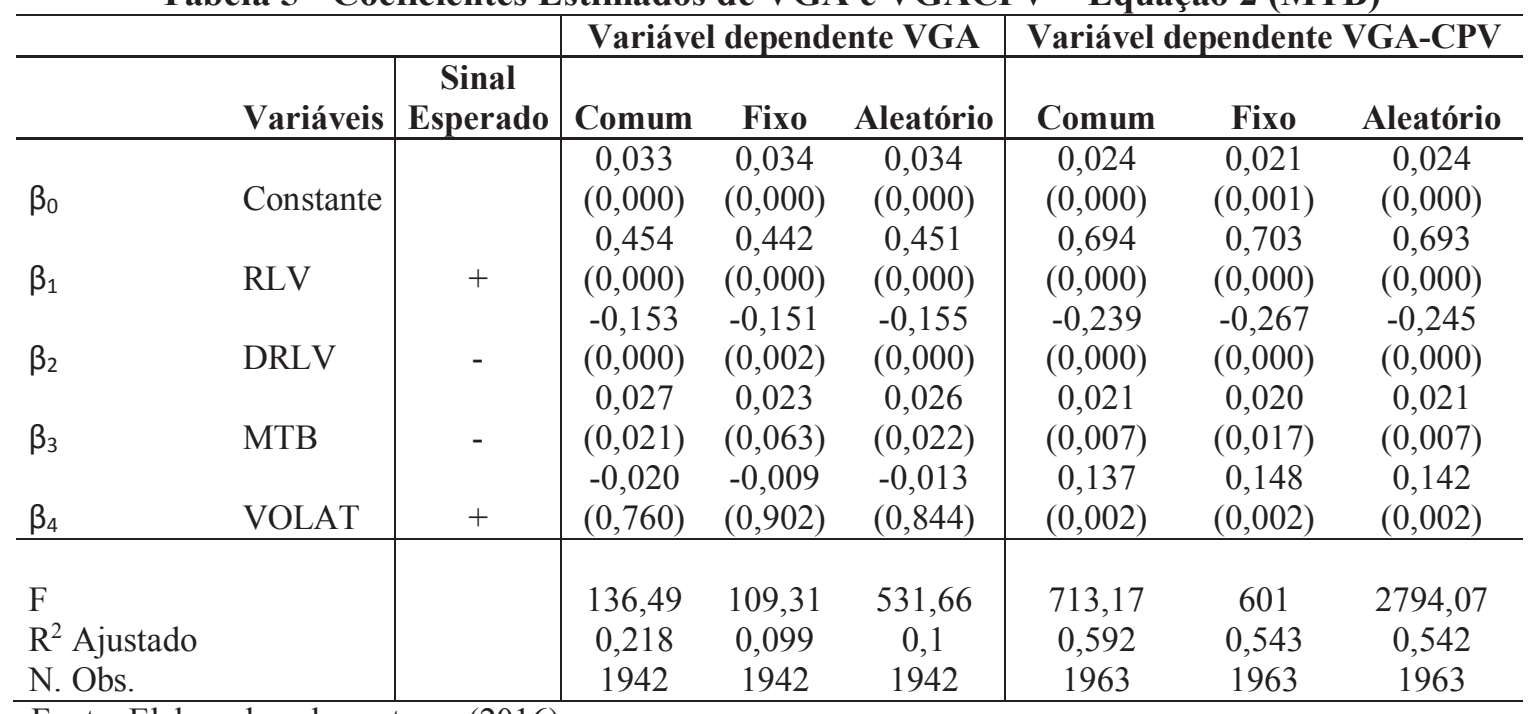

Fonte: Elaborada pelas autoras (2016).

O parâmetro positivo e significante de $\mathrm{B}_{3}$, tanto quando a variável dependente é VGA quanto para VGACPV, é contrário ao esperado, não permitindo aceitar a hipótese $\mathrm{H}_{2}$ do estudo. Esse resultado é contrário àquele encontrado por Anderson, Asdemir e Tripathy (2013). A relação market-to-book fornece uma indicação das expectativas de vendas de longo prazo. Uma possível explicação para a rejeição da hipótese $\mathrm{H}_{2}$ pode ser a dificuldade dos gestores das companhias brasileiras em planejar os recursos comprometidos, considerando as expectativas de crescimento das vendas no longo prazo. 


\section{Considerações Finais}

O objetivo desta pesquisa foi analisar se existe relação entre informações precedentes de vendas - crescimento histórico das vendas, a relação market-to-book e a volatilidade das vendas - e o comportamento assimétrico dos custos. A amostra da pesquisa é composta por 218 companhias abertas brasileiras com demonstrações financeiras publicadas no período 1995-2013. Os modelos econométricos testados nesta pesquisa são semelhantes àqueles utilizados por Anderson, Asdemir e Tripathy (2013); porém, além de testar os modelos com a variável dependente usualmente utilizada para avaliar a assimetria de custos (despesas de vendas, gerais e administrativas - VGA), os modelos também foram testados com as variáveis dependentes custos dos produtos vendidos e dos serviços prestados, somadas às despesas de vendas, gerais e administrativas.

Os resultados da pesquisa sugerem o comportamento assimétrico, tanto das despesas de vendas, gerais e administrativas (VGA), quanto destas despesas somadas aos custos dos produtos vendidos e dos serviços prestados (VGACPV). Porém, a assimetria de custos é maior para a variável VGA, sugerindo que os gestores conseguem gerenciar o CPV com mais facilidade quando da redução da receita. Adicionalmente, tem-se que o crescimento histórico das vendas, a relação market-to-book e a volatilidade das vendas possuem relação com a assimetria dos custos, sugerindo que informações precedentes de vendas podem afetar a gestão dos recursos comprometidos.

Como esperado, as companhias abertas brasileiras que apresentaram crescimento histórico das vendas mais persistente possuem menores níveis de assimetria de custos. No entanto, os gestores de empresas com histórico de volatilidade na receita líquida conseguem reduzir a assimetria dos custos dos produtos vendidos e serviços prestados, mas não conseguem essa mesma redução nas despesas de vendas, gerais e administrativas. Esses resultados diferem daqueles encontrados por Anderson, Asdemir e Tripathy (2013) para uma amostra de empresas americanas (origem common law), sugerindo que os gestores de países code law (como ocorre no Brasil) possuem dificuldades no planejamento na gestão estratégica de custos e a empresa apresenta volatilidade das vendas.

A relação market-to-book (MTB) apresentou relação positiva e significativa com as variáveis dependentes (VGA ou VGACPV), sugerindo que a assimetria de custos aumenta quando a MTB aumenta. Uma vez que essa relação fornece uma indicação das expectativas de vendas de longo prazo, uma possível explicação para essa relação direta pode ser a dificuldade dos gestores das companhias abertas brasileiras em planejar os recursos comprometidos e flexíveis, considerando as expectativas de crescimento das vendas no longo prazo.

Este estudo incorpora os custos dos produtos vendidos e dos serviços prestados na avaliação do comportamento assimétrico dos custos. Além disso, analisa a relação entre informações precedentes e os custos assimétricos no contexto brasileiro (país em desenvolvimento, code law e com ambiente econômico e político mais instável do que o americano). Esses resultados podem ser úteis para gestores analisarem como essas variáveis precedentes podem ser utilizadas na gestão estratégica de custos.

Para pesquisas futuras sugere-se analisar o horizonte temporal em que as informações precedentes podem afetar a assimetria de custos. Essa análise também pode ser realizada por setor de atividade e por tamanho de empresa. Além disso, os sinais das variações de custos

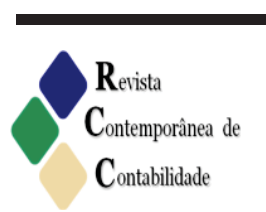

ISSN 2175-8069, UFSC, Florianópolis, v. 13, n. 28, p. 03-18, jan./abr. 2016 
também podem ser objetivo de investigação na análise da influência de informações precedentes nos stick costs.

\section{Referências}

ANDERSON, M.; ASDEMIR, O.; TRIPATHY, A. Use of precedent and antecedent information in strategic cost management. Journal of Business Research, v. 66, n. 5, p. 643$650,2013$.

ANDERSON, M. C.; BANKER, R. D.; JANAKIRAMAN, S. N. Are Selling, General and Administrative Costs "Sticky"?. Journal of Accounting Research, v. 41, n. 1, mar. 2003.

BANKER, R. D.; BYZALOV, D.; CHEN, L. T. Employment protection legislation, adjustment costs and cross-country differences in cost behavior. Journal of Accounting and Economics, v. 55, n. 1, p. 111-127, 2013.

CALLEJA, K.; STELIAROS M.; THOMAS D. C. A Note on Cost Stickiness: some international comparisons. Management Accounting Research, v. 17, n. 2, p. 127-140, jun. 2006.

COSTA, P. S. et al. Análise do Comportamento Assimétrico dos Custos nas Companhias Abertas dos Países da América Latina. Conferência Interamericana de Contabilidade, Uruguai, 2013. Disponível em: <http://www.cpcecba.org.ar/>. Acesso em: 05 fev. 2014.

DUARTE, S. L. et al. Comportamento das variáveis dos custos de produção da cultura do café no período de formação da lavoura. Revista Contabilidade Vista \& Revista, v. 24, n. 4, p. 15-33, out./dez. 2013.

KAMA, I.; WEISS, D. Do Earnings Targets and Managerial Incentives Affect Sticky Cost? Journal of Accounting Research, v. 51, n. 1, mar. 2013.

MARQUES, A. C. M. et al. Cost stickiness in Latin American open companies from 1997 to 2012. European Scientific Journal, may, p. 270 - 282, 2014.

MEDEIROS, O. R. COSTA, P. S., SILVA. C. A. T. Testes Empíricos Sobre o Comportamento Assimétrico dos Custos nas Empresas Brasileiras. Revista Contabilidade \& Finanças, São Paulo, v. 16, n. 38, mai./ago. 2005. Disponível em: <http://www.scielo.br>. Acesso em: 05 dez. 2013.

NOREEN, E. Conditions under which activity-based cost systems provide relevant costs. Journal of Management Accounting Research, p: 159 -168. 1991.

NOREEN, E.; SODERSTROM, N. Are overhead costs strictly proportional to activity?. evidence from hospital departments. Journal of Accounting and Economics, v. 17 n. 1-2, p: 255-278. 1994. 
NOREEN, E.; SODERSTROM, N. The accuracy of proportional cost models: evidence from hospital service departments. Review of accounting Studies, v. 2, p: 89-114, 1997.

SUBRAMANIAM, C.; WEIDENMIER M. L. Additional evidence on the sticky behavior of costs. Working Paper, 2003. Disponível em:

$<$ http://papers.ssrn.com/sol3/papers.cfm?abstract_id=369941> Acesso em: 28 jun. 2014.

VIA, N. D.; PEREGO, P. Sticky cost behavior: evidence from small and medium sized companies. Accounting and Finance, v. 54, n. 3, p. 753-778, abr. 2013. 